\title{
Study on Translation of the Names of Xi'an Snacks
}

\author{
Chunying Sui \\ Shaanxi Institute of International Trade \& Commerce, Xi'an, Shaanxi , China, 712046
}

Keywords: Xi'an snacks; translation; strategies

\begin{abstract}
With the development of the Silk Road economic belt, as well as the promotion of Silk Road heritage, Shaanxi Province will be more and more important in the process of internationalization. Therefore, the number of foreign tourists, business people and students will increase greatly. At the same time, the catering enterprises in Shaanxi Province will go abroad gradually, and Shaanxi snacks will be more and more popular in international market. However, there is no systematic research found in the translation of Shaanxi snacks till now. Therefore, this paper investigates the translation of Xi' an snacks after visiting major foreign-related hotels and some famous food streets near the attractions. Based on the investigation and analysis of the current situation of the translation of Xi'an snacks, this paper discusses the problems existing in the translation of Xi'an snacks, puts forward basic requirements for translation, and puts forward effective translation methods for dealing with existing problems. This study can make further contribution to the unification and standardization on the translations of Shaanxi snacks, spread the diet culture of Shaanxi dishes and help the foreign diners have a better understanding on characteristics of Shaanxi local culture.
\end{abstract}

\section{Introduction}

With the strengthening of China's opening up and exchanges, Chinese food culture is also going abroad, attracting more and more foreign friends. Food culture is a kind of national culture; therefore, the translation of food should be placed under the background of cultural exchange. That is to say, translation is not only the conversion of language, but conveys the connotation of Chinese dietary culture.

Shaanxi Province has a long history and profound culture, whose capital, Xi' an, is renowned at home and abroad of the world ancient civilization and international tourist city. For foreign tourists, Shaanxi's charm lies not only in the accumulation of Millennium culture and dotted historical sites, but in mouth-watering cuisine, such as stewed pancake in lamb soup, Chinese pork hamburger, cool noodles, etc., which attract tourists from all over the world. But the diverse translation versions of Shaanxi snacks names often make foreign guests confused, even misunderstand the real meaning of the snacks. Many of the translations include the following problems: taking the words too literally, inappropriate choice of words, long-winded, different translation versions, etc. This will undoubtedly affect the understanding of foreign visitors to Shaanxi snacks, and to a certain extent, lower the influence of Shaanxi food culture. Shaanxi snacks are the window and bridge for foreigners to understand the diet culture of Shaanxi province. Therefore, the translation of it is particularly important and it is very urgent to make Xi'an snacks translation more normative and prescriptive. The study on the translation of Xi'an snacks will be helpful for developing tourism industry and social economy of Xi'an, further promoting the city's external services, spreading the diet culture, and enhancing international influence and competitiveness of Shaanxi Province.

\section{Problems in Translation of Xi'an Snacks}

The author has investigated the status of Xi'an snacks translation, and the corpus sources include: The recipes in local restaurants of Xian such as Shangri-La Hotel, Sheraton Hotel, Nanyang International Hotel, Shaanxi Wen Yuan Hotel, yatailai, Huimin Street, etc.; The English Menus 
Service Manual of Classic Shaanxi Cuisine and Snacks issued by Shaanxi Provincial Department of Commerce; Chinese-English recipes of Chinese cuisine published at home and abroad; The official websites of Tourism Bureau of Shaanxi Province and Xian; Gourmet Club, such as the Daqin food, Taking you for $\mathrm{Xi}$ ' an snacks, etc.. Through the comparative analysis of the corpus, the author finds that there are very serious problems in the English translation of Xi' an snacks. Many people interpret the meaning of the snacks superficially, and consider neither the ingredients and spices nor the cooking methods of the dishes. Also the cultural connotation of some snacks did not be taken into account, and resulted in lots of jokes. The author summaries these problems appeared in the investigation in the following aspects:

\subsection{Capitalization problem}

English names on the menu should follow the rule that the first letter of content words should be capitalized while function words (for example, conjunctions, and prepositions) needn't. It is, however, a very common phenomenon for many star hotels to make such kind of mistakes on the snack translation. Typical examples in order are as follows. First, a Xi'an famous snack "Nong Jia Jiao Tuan" is translated into "stir farm group" in certain hotel. Without considering whether its translation is better or not and commenting on its accuracy, only from the English name can we see the silly mistake it made, which means that it did not follow the capitalization rules. Thus, it should be "Stir Farm Group". As another example, on the menu of a certain hotel "Qian Ceng Guo Kui" is interpreted as "melaleuca daguokui". This snack is a local star hotel's signature snack, and is placed in the most prominent place without being aware of this capitalization problem. So it should be changed into "Melaleuca Da Guo Kui". Capitalization problem is such a simple error which is caused by the carelessness of the translator or the staff who failed to notice it. Only if they pay a little attention on it or make the translations to be amended can such mistakes be avoided.

\subsection{Lack of English translation version}

In 2007, the Shaanxi Provincial Department of Commerce formally published the English names of 50 kinds of Shaanxi snacks with Chinese Business News jointly, and recommended it to the restaurants, hotels and other service institutions to promote the use of such bilingual menus. But this bilingual menu contains only part of Shaanxi snacks, and others are not included in, such as a famous snack in Shaanxi "Biang Biang Mian". In addition, as a famous place of historical interest, Bell Tower is the symbol of Xi'an, but there are only Chinese menus in the foreign hotels nearby, including Tong Shengxiang and De Fachang. As an attractive place for foreign tourists, the lack of English translation of Shaanxi sancks there really makes people shocked and unbelievable.

\subsection{Lack of standard}

Snack translation belongs to the category of practical English, and many expressions of Chinese snacks can be found in the corresponding English language. However, there are also some non-corresponding cases commonly appeared in the translation of Chinese and English languages. It is regrettable that, so far, only Beijing, Shanghai, Guangzhou and other cities with a higher degree of internationalization have made a quest for the standardization requirements. Shaanxi Provincial Department of Commerce also has issued the first standard of English translation of Shaanxi snacks. However, it has been proved that this standard has not yet been fully used according to the results of this survey. For example, for a same Shaanxi snack "Qi Shan Sao Zi Mian", there are various translation versions about it in different hotels. Some hotels translate it into "qishan sao surface", while others translate it as "sour face", and so on. In addition, the translations of the same snack on different medias such as Shanxi travel magazines, travel websites, newspapers for cultural promotion, etc. are all not consistent. As a typical representative of the diet culture, snack is not only an important part of tourism culture, but it can better reflect local life style and traditional food culture compared with other dishes which have been influenced greatly by foreign culture. Therefore, it is really an important part of developing Shaanxi tourism industry to make the translation of Xi'an snacks more normative and foreign visitors really clear about the charm and culture of Xi' an diet 
cultures.

\subsection{Insufficient information}

The English translation versions of some snacks are too general, and the information expressed is insufficient, which is unable to reflect the characteristics of the foods. Taking "Qishan Sao Zi Mian" again as an example, it has more than 3,000 years of history, and a good reputation for its fine workmanship, excellent ingredients, color, aroma, shape and taste. Nowadays, however, its English name "Qishan noodles" is no use for foreign guests to understand the characteristic of this snack, because the English version is too general. Although the name is showed in English, foreign visitor could not understand what is the difference between this noodle and other noodles, what kind of raw materials it has and what is its distinctive feature when they see it at the first sight. Thus, according to its materials and shape of ingredients, "Qishan Sao Zi Mian” might be translated into "Noodles with Diced Pork, Qishan Style", which could be helpful for foreigners to learn about its features in detail.

\section{Reasons of the Problems}

The study on snacks translation is a new research area, so it is normal to be chaotic. During the survey, the author found many translation problems of Shaanxi snacks and the causes of them by conducting the questionnaires, interviews and searching for relevant information through other ways. What makes the author sad is that most people pay little or no attention on the feeling of the readers, namely, the foreign guests in snack translation, which becomes the internal reason of these problems. There are also some external reasons which are mainly shown in the following aspects after summing up by the author:

\subsection{Ignorance of the importance of snacks}

As an auxiliary of the main meal, snacks are often put in the last part of the menu in many star hotels in Shaanxi. People put far less emphasis on the translation of snacks than to the main dishes. However, what gives foreign tourists deep impression when they visit the different food courts and food streets in the cities is the unique flavor of snacks, which make them indulged in pleasures without stop. Therefore, snacks and main dishes are equally important.

Besides, related functional departments of our government did not realize the seriousness of the problem as well as possible adverse effects for a long time. They didn't have a unified supervision to the translation of snack names to make them more normative and acceptable. All the functional departments decide on their own that whether they should translate the snack name, how to translate it and is translated by whom. So the most direct result of this is one snack name with several English versions. In addition, it related to the cost to replace the wrong snack versions, and that is also a factor that prevents the translation of Shaanxi snacks from normative.

\subsection{Lack of relevant research}

Current research on English translation of Chinese dish names is very haphazard and accidental, which is lack of guidance and standards. Snacks translation is brought into the study of tourism texts by translators in China. Although there are few studies on it which are showed by paper, there are still lack of specialized, comprehensive research system, and no specialized books are published at present. Through searching and making inquires in some major periodicals and on the website, the author finds that there are fewer researches on the translation of Xi'an snacks currently. Because there are great differences between the Eastern and Western cultures, raw material of foods, and cooking methods, the translation of Xi'an snack names is not so simple as imagined, and the most important factors affecting the translation of snacks are cooking method and the way snacks named. When translating, the translators should pay more attention to these two factors and think about how to combine them so as to make the English version of snacks not only reflect the Shaanxi characteristics, but conform to the naming conventions of Western cuisine. Thus, all these factors 
make the translation of snack names very difficult.

\section{Translation Strategies}

The essence of snack texts is to deliver a kind of communication information to the audience of the diet texts under certain context, hoping to achieve specific perlocutionary effects, that is, giving expression to the communicative intention of diet text users. From this point, a snack text is a communicative behavior. The publisher of a snack text is the addresser, the audience of a snack text is the addressee, and the service environment of the snack text is the context of situation. There is no doubt that the translation of the snack text is a cross-cultural communicative activity. As a communicative behavior, the translation of the snack text is a kind of "one-way communication", and both parties involved in the communication have no opportunity to discuss and exchange information; the addressee's understanding of the communicative discourse can only rely on the existing text and the specific context that the text relies on. As a result, to make sure of the success of this cross-cultural communication, the author believes that in translating snack texts, translators are supposed to make sure that the target language is supposed to be simple and easy to understand, the content and format should accord with the codes of the target language and it also needs to realize the pragmatic effect of the translation.

\subsection{Snacks named by the cooking method}

The translation of the snacks focusing on cooking stresses the cooking method of snacks. In the field of snacks, the commonly used cooking methods include "frying, deep frying, roasting, stir-frying". Such cooking methods mainly stress the "smell” of snacks. Therefore, in translating such snacks, the translation principle takes cooking as the principal thing and the main ingredient as the subsidiary role. Translating the cooking method and raw materials of snacks can arouse foreigners' appetite and make them product the desire of understanding and tasting.

\subsection{Snacks named by the snack's shape or taste}

The translation of such snacks usually attracts tourists with their shapes by creating a beautiful visual enjoyment so as to attract tourists to taste. Therefore, in translating this type of snacks, it is necessary to make their shapes stand out. The author believes that in translation, we can use the straightaway keyword "shape" to directly manifest the shape of the snacks.

\subsection{Snacks similar to some western dishes}

Names of Xi'an snacks are usually possessed of distinct Shaanxi local features. Such local features sometimes can't find equivalent words in mandarin, not to mention in a foreign language. However, English snack names with distinct local features are difficult to bring foreign tourists intimacy and can't arouse their desire of consumption. Therefore, in translating Xi'an snacks, if it is possible to find words with similar cooking methods or shapes in English, it will be unnecessary to find other ways and translators can directly use these words for translation.

\section{Conclusion}

Because of the important role that Xi'an snacks play in Shaanxi diet culture, their translations are very necessary in the situation of economic globalization. Translators should know that the translation of Xi'an snacks will have a great influence on the development of Shaanxi diet culture and the transmission of Chinese culture. Nowadays, more and more foreign guests have come to Xi'an to work, live, study or travel, and it has become a necessary part for them to taste the unique and special Xi'an snacks. As an important way of transmitting culture, Xi'an snacks are delicious and unique, which possess certain significance in external exchanges of diet culture. Thus, the improper English version of Xi'an snack names not only can correctly transmit Shaanxi diet culture, but also greatly influence foreigners' appetite, even cause negative influence to Shaanix's tourism. Translating Xi'an snack names is also a kind of external publicity. To some extent, standard and 
authentic translation of Xi'an snack names can reflect a city's level of civilizations; the broadly application of English version of Xi'an snack names is a sign of level of openness of a city; the standard application of English version of Xi'an snack names is an inspection of the level of the city's degree of internationalization; and proper translation of Xi'an snack names is a reflection of a city's overall quality. Therefore, the author has launched the study on translation of Xi'an snack names.

\section{References}

[1] Zong Lijuan. Translation on the name of Shaanxi snacks from the perspective of Skopos theory[J]. Intelligence, 2013(26).

[2] Tian Lili, Tian Zhiqiang. On the English translation of characteristic snacks [J]. Journal of Baoji University of Arts and Sciences, 2013(3).

[3] Wu Guodong. Xi'an characteristic snack Guide [M]. Xi'an: Xi'an Publishing House, 2007. Ren Jingsheng. English translation on Chinese vegetables and staple food [J]. Chinese Translation Journal, 2001(6):22.

[4] Nida, Eugene A. \&Taber, Charles R. The Theory and Practice of Translation [M]. Shanghai: Shanghai Foreign Language Education Press, 2004. 